\title{
Dysphagia as the Sole Initial Presentation of Celiac Disease
}

\author{
Andrew C. Berry ${ }^{1, *}$, Rahman Nakshabendi ${ }^{2}$, Ozdemir Kanar ${ }^{2}$, Hussein Abidali ${ }^{3}$, Ahmad Nakshabandi ${ }^{4}$, \\ Aaron C. Baltz ${ }^{5}$, James T. Kwiatt \\ ${ }^{1}$ Department of Medicine, University of South Alabama, Mobile, AL, USA \\ ${ }^{2}$ Department of Medicine, University of Florida College of Medicine-Jacksonville, Jacksonville, FL, USA \\ ${ }^{3}$ Department of Medicine, Banner Good Samaritan Medical Center, Phoenix, AZ, USA \\ ${ }^{4}$ Tampa General Hospital, Tampa, FL, USA \\ ${ }^{5}$ GI Associates, Wauwatosa, WI, USA \\ *Corresponding author: Aberry5555@gmail.com
}

Received July 17, 2015; Revised July 27, 2015; Accepted July 28, 2015

\begin{abstract}
Several studies have described celiac disease patients presenting with esophageal symptoms. We present the case of a 33-year-old Caucasian woman who presented with three months of strictly dysphagia to solids. She reported no weight loss, abdominal pain, or diarrhea and reported no heartburn symptoms. Labs revealed a tissue transglutaminase IgA of $>100$ units. Biopsies revealed moderate to severe villous blunting and increased intraepithelial lymphocytosis consistent with Marsh 3 celiac disease in the $2^{\text {nd }}$ and $3^{\text {rd }}$ portions of the duodenum. To the best of our knowledge, this case represents the first reported case of dysphagia as initial and sole presentation of celiac disease completely resolved by gluten-free diet.
\end{abstract}

Keywords: dysphagia, celiac disease, esophagogastroduodenoscopy, endoscopy

Cite This Article: Andrew C. Berry, Rahman Nakshabendi, Ozdemir Kanar, Hussein Abidali, Ahmad Nakshabandi, Aaron C. Baltz, and James T. Kwiatt, "Dysphagia as the Sole Initial Presentation of Celiac Disease.” International Journal of Celiac Disease, vol. 3, no. 3 (2015): 108-109. doi: 10.12691/ijcd-3-3-5.

\section{Introduction}

Celiac disease patients typically present with common symptoms such as abdominal pain and discomfort, bloating, diarrhea, constipation, and irritable bowel-like symptoms, but can also present with extra-intestinal complaints [1,2]. Several studies have described celiac disease patients presenting with esophageal symptoms [3]. Celiac disease is loosely linked with eosinophillic esophagitis and Plummer-Vinson Syndrome [4]. Both of these conditions are known culprits of dysphagia [4]. Due to these factors, dysphagia is thus considered a symptom of celiac disease.

\section{Case Report}

A 33-year-old Caucasian woman with no significant medical history presented with three months of dysphagia to solids. She had "tightness" in her chest with eating bananas. She reported no weight loss, abdominal pain, or diarrhea. She had no heartburn symptoms, although reported a two-year history of mild heartburn six years prior, which was successfully treated with once daily proton pump inhibitor (PPI) for 24 months. She also did not have any allergic rhinitis symptoms. There was no family history of eosinophilic esophagitis, inflammatory bowel disease, upper gastrointestinal cancers, or celiac disease. Due to her persistent dysphagia, an esophagogastroduodenoscopy (EGD) was performed revealing mild bulbar duodenitis, and scalloping in the 2nd and 3rd portions of the duodenum. Biopsies were taken from her mid esophagus, distal esophagus, stomach antrum, and $2^{\text {nd }}$ and $3^{\text {rd }}$ portions of the duodenum. Labs revealed a tissue transglutaminase IgA of $>100$ units. Serum IgA, vitamin A, folic acid, Vitamin B12, Vitamin $\mathrm{D}$, thyroid stimulating hormone, iron studies, complete blood count, complete metabolic panel, and Helicobacter pylori IgG antibody were all within normal limits.

Biopsies revealed no abnormalities in the mid esophagus, mild reflux esophagitis in the distal esophagus, mild gastritis with no evidence of $H$. pylori in the stomach antrum, and moderate to severe villous blunting and increased intraepithelial lymphocytosis consistent with Marsh 3 celiac disease in the $2^{\text {nd }}$ and $3^{\text {rd }}$ portions of the duodenum. Her esophagus was empirically dilated with a 45, then 48, then 51 French Savary dilator over a guidewire. Her dysphagia persisted post dilation. The patient was started on a gluten-free diet. Three months after starting the gluten-free diet her dysphagia resolved. Follow-up tissue transglutaminase IgA at the time of resolution of the dysphagia was undetectable indicating resolution of the celiac disease. This patient presented with solid food dysphagia unresponsive to empiric dilation; dysphagia resolved with a gluten-free diet and correlated to serologic resolution of disease. This suggests her newly-diagnosed celiac disease was responsible for her dysphagia. 


\section{Discussion}

To the best of our knowledge, this case represents the first reported case of dysphagia as initial and sole presentation of celiac disease completely resolved by gluten-free diet. This patient did not have evidence of eosinophillic esophagitis, Plummer-Vinson Syndrome, or any other culprit for her dysphagia. She also did not complain of any other typical celiac disease symptoms, such as abdominal pain and discomfort, bloating, diarrhea, constipation, and irritable bowel-like symptoms [1,2]. The patient's dysphagia continued after the endoscopy for 3 months despite the dilation. Laboratory testing of elevated tissue transglutaminase IgA level, followed by gluten-free diet with subsequent resolution of symptoms and normalization of tissue transglutaminase IgA confirmed the patient's celiac disease course.With introduction of the gluten-free diet, the dysphagia resolved. By the time her tissue transglutaminase IgA level had normalized three months post diagnosis, the dysphagia had completely resolved as well. Antibody normalization and the lack of symptoms correlated.

Given that the only know treatment for celiac disease, gluten-free diet, led to resolution of her symptoms, one would postulate that the dysphagia and active celiac disease were related. Re-introduction of gluten would result in recurrence of the dysphagia. Given that the gluten-free diet did not immediately improve the symptoms until the tissue transglutaminase normalized, it is likely the active celiac disease itself that caused the symptoms, and not immediate ingestion of gluten.

The patient's symptoms were also present with things like bananas (gluten-free), suggesting that long-term intestinal damage played a role in the dysphagia. The mechanism by which celiac disease causes dysphagia is still unknown. Possible mechanisms for celiac causing dysphagia could include a neuropathy causing either esophageal dysmotility or visceral hypersensitivity in the upper gut. Another potential mechanism may involve subclinical nutritional deficiencies. As gluten-free diets are anecdotally being used for treatment of other neurologic conditions such as migraines, and gluten-free diets have also been known to resolve many gastrointestinal symptoms in patients aside from diarrhea, this case illustrates the need to explore the pathology of celiac disease beyond the duodenum.

Celiac disease is a diagnostic consideration in the evaluation of unexplained dysphagia. In patients presenting with solid food dysphagia, EGD remains the exam of choice. Although it would be academically interesting to see a before and after esophageal manometry performed, there would be no clinical benefit to the patient. Whether celiac disease should be routinely excluded in patients with dysphagia warrants further study.

\section{Conclusions}

Celiac disease is loosely linked with eosinophillic esophagitis and Plummer-Vinson Syndrome, culprits of dysphagia and thus dysphagia can be considered a symptom of celiac disease. However, dysphagia as the sole initial presentation of celiac disease is very uncommon

\section{Conflict of Interest}

None of the authors have any conflicts of interest to disclose. None of the authors listed received any financial support or services or any other contributions for their work. All patient identifiers have been removed.

\section{References}

[1] Rubio-Tapia A, Hill ID, Kelly CP, Calderwood AH, Murray JA. American College of Gastroenterology ACG clinical guidelines: diagnosis and management of celiac disease. Am J Gastroenterol 2013; 108: 656-676.

[2] Rastogi A, Bhadada SK, Bhansali A, Kochhar R, Santosh R. Celiac disease: a missed cause of metabolic bone disease. Indian $J$ Endocrinol Metab. 2012; 16: 780-785.

[3] Iovino P, Ciacci C, Sabbatini F, et al. Esophageal impairment in adult celiac disease with steatorrhea. Am J Gastroenterol. 1998; 93 1243-1249.

[4] Lucendo AJ. Esophageal manifestations of celiac disease. Diseases of the Esophagus. 2011; 24: 470-475. 